S.D. Ryder, D.J. Pisano, M.A. Walker, and K.C. Freeman, eds.

\title{
Bulge Formation in Late-type Galaxies - Cuspy- vs Soft-core Dark Matter Density Profiles
}

\author{
Huang, J.-H \\ Department of Astronomy, Nanjing University, Nanjing, China
}

Deng, Z.-G

Department of Physics, Graduate School, CAS, Beijing, China

$\mathrm{Fu}, \mathrm{Y} . \mathrm{N}$

\section{Purple Mountain Observatory, CAS, Nanjing, China}

\begin{abstract}
Observational data increasingly indicate that the mergers of galaxies, even minor mergers, can trigger the formation of a set of SSCs within galaxies. The follow-up, dynamical evolution of SSCs in a configuration of dark matter (DM) dominated systems would be basically similar to what we simulated in this work. The scenario that we propose yields a series of results comparable to the relevant observations. Most notable are two points. One is that the low bulge detection rate in very late-type galaxies is in favour of soft-core DM profiles in galaxies of this kind. On the other hand, the observed young ages of nuclear clusters provide evidence supporting cuspy-core profiles. In conclusion, both the soft- and cuspy-core DM density profiles are suitable to relevant dwarf galaxies based on comparisons with observations.
\end{abstract}

The dynamical evolution of super star clusters (SSCs) as a bulge formation model (Fu, Huang, \& Deng 2003, MNRAS, 339, 442) is investigated in dark matter halos depicted with the NFW or the Burkert density profile. The simulations show that:

(1) exponential bulges with central cusps form in both cases, however, distinctive bulge formation rates are derived for the NFW and the Burkert profiles, as shown in Fig 1. Compared with the high bulge formation rates of $97-100 \%$ at ages of 1-4 Gyrs in the NFW case, the rate derived in the Burkert profile becomes as low as $30-70 \%$, shown in the right panel of Figure 1.

(2) masses of the formed bulges are causally connected with masses of nuclear clusters, fully accordant with Balcells et al's work (2003, ApJ, 582, L79). The formed bulges and nuclear cusps are heavier in the case of a cuspy-core profile. (3) massive, nuclear star clusters possibly form with no discernible bulges at ages of about $1 \times 10^{7} \mathrm{yr}$ and $1 \times 10^{8} \mathrm{yr}$ in the NFW and Burkert cases, respectively, as indicated in Fig 3.

(4) based on comparisons with observations, both the soft- and cuspy-core dark matter density profiles are suitable to relevant dwarf galaxies. 

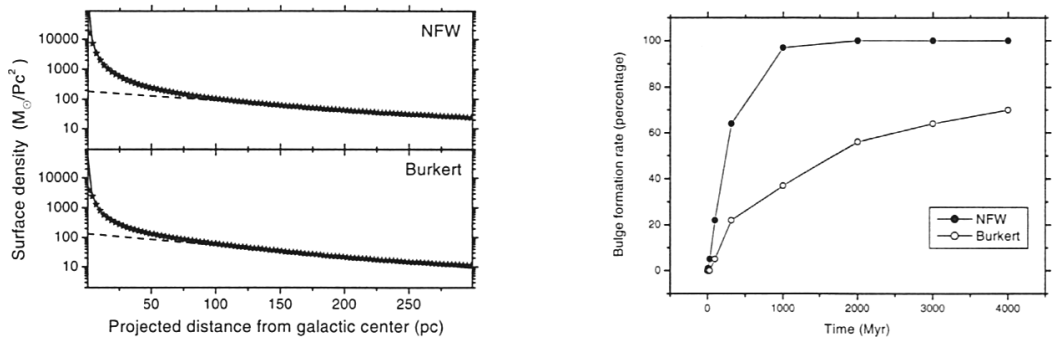

Figure 1. Left panel indicates the mean surface density profiles of the formed bulges at $3 \mathrm{Gyr}$, along with the curves depicting the model fitting. Dashed lines are fitted exponential components, solid lines are fits to (Exp. + cusp) model. The right panel is self-explanatory.

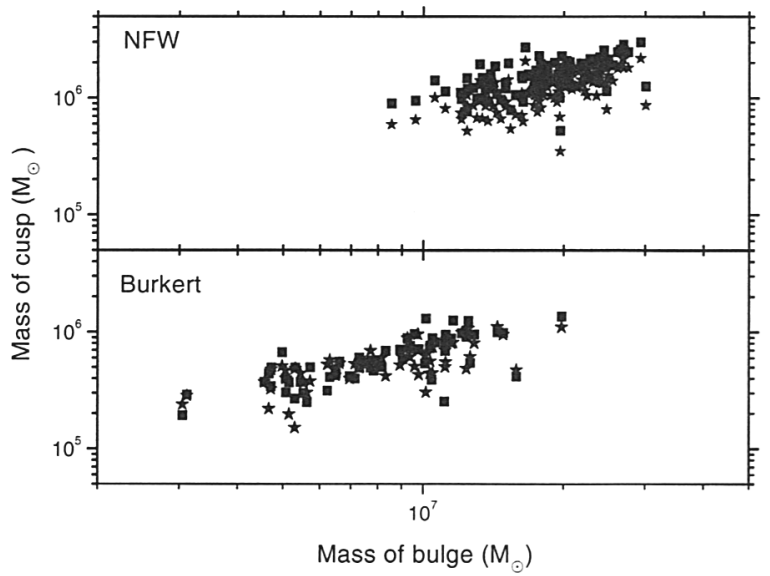

Figure 2. Predicted mass relation of the formed bulges to their central cusps. The filled squares and stars denote masses of central cusps obtained by spatial resolution of 2 and 5 pc, respectively.

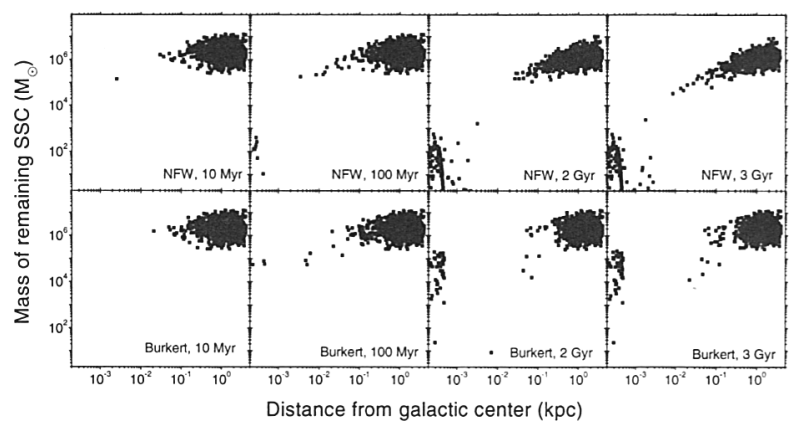

Figure 3. Mass of remaining SSCs of simulated galaxies $v s$ the SSCs' distance from the halo centre in the NFW and Burkert halos. 